\title{
Germanica
}

\section{Hans Esselborn, Die Erfindung der Zukunft in der Literatur. Vom technisch-utopischen Zukunftsroman zur deutschen Science Fiction}

\section{Françoise Willmann}

\section{(2) OpenEdition}

Journals

Édition électronique

URL : https://journals.openedition.org/germanica/9216

DOI : 10.4000/germanica.9216

ISSN : 2107-0784

\section{Éditeur}

Université de Lille

Édition imprimée

Date de publication : 1 juin 2020

Pagination : 198-201

ISBN : 978-2-913857-45-2

ISSN : 0984-2632

\section{Référence électronique}

Françoise Willmann, « Hans Esselborn, Die Erfindung der Zukunft in der Literatur. Vom technischutopischen Zukunftsroman zur deutschen Science Fiction », Germanica [En ligne], 66 | 2ème trimestre 2020, mis en ligne le 01 janvier 2021, consulté le 08 janvier 2022. URL : http://

journals.openedition.org/germanica/9216; DOI : https://doi.org/10.4000/germanica.9216

Ce document a été généré automatiquement le 8 janvier 2022.

(c) Tous droits réservés 


\title{
Hans Esselborn, Die Erfindung der Zukunft in der Literatur. Vom technisch-utopischen Zukunftsroman zur deutschen Science Fiction
}

\author{
Françoise Willmann
}

\section{RÉFÉRENCE}

Würzburg, Königshausen \& Neumann, 2019, 427 p.

1 L'ouvrage de Hans Esselborn n'est pas une histoire de la science-fiction allemande. Ce serait doublement vain, d'une part parce que la science-fiction comporte de multiples sous-genres, fantasy, cyberpunk, space-opera etc., et d'autre part parce qu'elle ne saurait être détachée, ni du terreau, ni des interférences voire des hybridations avec les productions du même type d'autres aires linguistiques et culturelles. L'étude d'Esselborn se focalise sur la littérature, essentiellement sur le roman, et suit un fil directeur, le concept d' "esthétique du futur ». C'est dans cette perspective qu'il en replace l'émergence et l'évolution entre futurologie et utopie, entre mise en scène du progrès et crainte du déclin, dans des univers étrangers ou parallèles où évoluent des êtres dont l'altérité nous divertit et nous questionne.

2 Pour tenter d'en cerner les contours, Esselborn analyse diverses tentatives de définition et s'appuie notamment sur Darko Suvin dont il retient les notions désormais classiques de « cognitive estrangement » et de «novum ». La synthèse qu'il en dégage fonde sa propre démarche :

La science-fiction est le déploiement narratif d'une pensée du possible, d'une manière cohérente et méthodique qui exige et permet une explication rationnelle. 
En partant d'un moment nouveau marquant, généralement installé dans le futur, d'un « novum » fait de technique, de science et de société, on construit des mondes alternatifs, des sociétés futures et des êtres étrangers qui se distinguent fondamentalement de l'environnement empirique de l'auteur, tout en y renvoyant implicitement. L'univers textuel sert à mettre le présent à distance en sorte de favoriser la connaissance [...], en fait un modèle qu'on peut comprendre et critiquer. (p. 54)

3 De la sorte, il caractérise ce qui, dans la science-fiction, tient de la connaissance (et, remarque-t-il en commentant Suvin, ne concerne pas les $90 \%$ de productions textuelles du genre qui relèvent de la paralittérature). Mais la science-fiction est d'abord littérature, et en tant que telle, il convient de ne pas négliger sa double fonction, qui est à la fois de distraire et de délasser tout en permettant au lecteur de s'orienter dans un monde en perpétuelle transformation, voire de s'y accoutumer. Et en tout état de cause, ces lignes directrices ainsi dégagées ne cessent d'être remises en question par les œuvres elles-mêmes qui se jouent des limites, empruntent à toutes les tendances voire à tous les médias (cinéma, jeu vidéo), font de l'intertextualité une pratique d'écriture privilégiée. Le recours à la perspective historique fournira à l'auteur un principe d'ordre supplémentaire, ainsi que la tentative de dégager thèmes et motifs récurrents, parmi lesquels les voyages interstellaires, les rencontres avec les aliens (qui ne sont plus seulement des extraterrestres), l'intelligence artificielle, etc.

4 À tout seigneur tout honneur : c'est avec un chapitre consacré à Jules Verne que commence la partie où l'on entre dans le vif du sujet, à travers l'analyse de textes marquants. En réalité, c'est une triade qu'Esselborn place aux origines de la sciencefiction allemande : Jules Verne (1828-1905), Herbert George Wells (1866-1946) et Kurd Lasswitz (1848-1910), ce dernier moins célèbre que le Français et l'Anglais, son roman phare, Auf zwei Planeten, n'ayant pas été traduit en français et seulement tardivement en anglais. Esselborn tente de dégager ce qui en fait selon lui les fondateurs du « roman utopique et technique » avant la première guerre mondiale. Pour chacun d'eux, il met les fils conducteurs retenus à l'épreuve d'une lecture rapprochée d'œuvres précises en entretenant également le dialogue avec la littérature critique. C'est ainsi qu'il distingue le roman d'aventure à la Jules Verne, l'imaginaire centré sur la technique de Bernhard Kellermann (1879-1951) dont il analyse le célèbre roman Der Tunnel (1913), et les projections astrales poétiques d'un Paul Scheerbart (1863-1915). Une nouvelle étape, l'entre-deux-guerres, verra le succès de Hans Dominik (1872-1945), élève de Lasswitz, mais nullement son disciple. Il convenait de donner toute sa place à Dominik pour plusieurs raisons: sa réception qui s'est prolongée jusqu'au $\mathrm{xx}^{\mathrm{e}}$ siècle (il est toujours réédité), sa réputation ternie par les nombreuses critiques lui reprochant nationalisme, racisme, voire fascisme (une accusation contre laquelle Esselborn produit des arguments convaincants), enfin sa version d'une littérature d'anticipation technique qu'on peut opposer à la direction empruntée par Kurd Lasswitz, au point que Esselborn identifiera par la suite une tendance de la science-fiction dans la lignée de ce dernier et dans laquelle il inclut également Alfred Döblin (1878-1957), avec Berge Meere und Giganten $(1924,1932)$. On discerne jusqu'à nos jours les traces de ce double héritage, le roman d'anticipation optimiste, fondé sur la foi dans le progrès scientifique et technique et sa dimension éthique, porté par l'esprit de Lasswitz, et le roman d'aventure à suspense fondé sur les réussites techniques, l'héritage de Verne et de Dominik. 
5 Après la Seconde Guerre mondiale, un thème nouveau s'impose : l'énergie atomique, et plus précisément la bombe atomique, occupent la place centrale dans les innovations techniques qui engagent le futur, nourrissant les projections dystopiques, voire les interrogations sur l'avenir même de l'humanité. Arno Schmidt (1914-1979), un auteur culte des amateurs de science-fiction, nourri de Verne et de Lasswitz autant que de littérature anglo-saxonne, reprend la menace technologique sur fond de guerre froide et de pessimisme anthropologique débouchant sur la plausibilité d'une autodestruction de l'humanité. D’Ernst Jünger (1895-1998) on connaît la fascination pour la technique. Esselborn retient surtout Heliopolis $(1949,1964)$, pour en étudier les différentes facettes résistant à toute interprétation univoque.

Durant quelques décennies, le genre de l'anticipation technoscientifique connaît une spécificité allemande du fait de l'existence de la RDA. À l'écart de l'influence américaine, imprégnée de l'idéologie du progrès social et technique et marquée par les succès de la recherche spatiale soviétique, la «littérature utopique " du socialisme réellement existant s'est largement conformée aux exigences d'une écriture réaliste et conventionnelle. Si Esselborn laisse entendre que cette littérature pèche souvent par manque d'audace, il n'en note pas moins l'existence d'un certain nombre d'auteurs qui surent sortir des sentiers battus. C'est le cas notamment de deux couples, Johanna Braun (1929-2008) et Günter Braun (1928-2008), qui écrivirent ensemble tous leurs textes, et Angela Steinmüller (*1941) et Karlheinz Steinmüller (*1950), auteurs également de textes critiques.

7 Cependant, le passage du roman d'anticipation à la science-fiction à proprement parler nous ramène aux Etats-Unis, avec les célèbres Isaac Asimov (1920-1992), Philip K. Dick (1928-1982) et William Gibson (*1948): la science-fiction allemande, tout en restant redevable aux grands précurseurs, prendra modèle sur ces auteurs américains et bien d'autres. Entrent dans les récits du futur les robots, les mondes virtuels et parallèles, les cyberespaces, et autres clones, androïdes ou cyborgs. Et c'est dans les années $1960 \mathrm{du}$ $\mathrm{xx}^{\mathrm{e}}$ siècle que Esselborn voit se constituer une véritable science-fiction allemande, qu'il resitue dans son contexte éditorial, avec l'implication de Goldmann, Heyne, et même, un temps, Suhrkamp. La dernière partie du volume propose des analyses d'ouvrages marquants des auteurs contemporains les plus reconnus: Herbert W. Franke $\left({ }^{*} 1927\right)$, Carl Amery (1922-2005), Wolfgang Jeschke (1936-2015), Andreas Brandhorst (*1956), Andreas Eschbach (*1959), Frank Schätzing (*1957), Hans Joachim Alpers (1943-2011), Michael Marrak (*1965), Uwe Post (*1968), Marcus Hammerschmitt (*1967), Benjamin Stein $\left({ }^{*} 1970\right)$ et enfin Dietmar Dath $\left({ }^{*} 1970\right)$.

On l'aura compris : l'ouvrage de Hans Esselborn est d'une grande richesse, tant du point de vue des textes qu'il étudie, auteurs de science-fiction et critiques du genre. Il réussit à en donner une vue d'ensemble qui, malgré ses dimensions contraintes, échappe à l'écueil du survol. Dans la profusion des textes étudiés, il constate pour finir la consécration de la tendance à la narration ludique, soucieuse surtout de divertir, face à la dimension spéculative voire philosophique privilégiant l'expérience de pensée. L'enjeu délicat de la science-fiction serait sans doute de faire coïncider l'expérience de pensée, l'imagination prospective, et une écriture qui ne se contente pas des recettes efficaces du roman d'aventure. Il semblerait que Hans Esselborn considère que Dietmar Dath est actuellement l'auteur qui réalise au mieux cette synthèse si délicate, mais son ouvrage fournit bien d'autres pistes encore aux lecteurs et lectrices prêts à découvrir un genre qui mérite décidément qu'on lui accorde davantage d'intérêt. 